International Mathematical Forum, 2, 2007, no. 39, 1945 - 1956

\title{
Backward r-Difference Operator and Finding Solution of Nonhomogeneous Difference Equations
}

\author{
Hassan Hosseinzadeh and G. A. Afrouzi ${ }^{1}$ \\ Islamic Azad University, Ghaemshahr Branch \\ P.O. Box 163, Ghaemshahr, Iran
}

\begin{abstract}
In this paper we introduce a new operator that we call it the backward $r$-difference operator $\nabla_{r}$ which is defined in the following:

$$
\nabla_{r} y_{n}=y_{n}-r y_{n-1}
$$

Then, we investigate some properties of this new operator, we find a shift exponential formula and use it for finding solution of the nonhomogeneous difference equations with constant coefficients, may be written in the following form

$$
\left(\prod_{i=1}^{m} \nabla_{r_{i}}\right) y_{n}=f_{n} .
$$
\end{abstract}

Keywords: Backward difference operator $\nabla$; backward $r$-difference operator $\nabla_{r}$; Difference equation; Shift exponential operator; Particular solution

\section{Preliminaries}

1.1. In Numerical Analysis, we use some linear operators, those are shift exponential operator $E$, " $E f_{j}=f_{j+1}$ ", Backward difference operator $\Delta$, " $\Delta f_{j}=$ $f_{j+1}-f_{j}$ " and backward difference $\nabla, " \nabla f_{j}=f_{j}-f_{j-1}$ ". These operators are used in some topics of Numerical Analysis, particularly in interpolation, quadratures, difference equations, and so forth see [1], [2], [4].

Since $E, \Delta$ and $\nabla$ are linear operator, in addition to every order of them, their inversion $E^{-1}, \Delta^{-1}, \nabla^{-1}$ and every polynomial of them are linear too. We know that the difference equation appears in numerical solution of ODE, PDE, IE, IDE, .

\footnotetext{
${ }^{1}$ Corresponding author E-mail: afrouzi@umz.ac.ir also both authors are in : Department of Mathematics, Faculty of Basic Science, Mazandaran University, Babolsar, Iran.
} 
In this paper we find particular solution of nonhomogeneous difference equations with constant coefficients. The difference equations are written in one of the following forms:

Under the shift operator $E$

$$
\begin{aligned}
& P(E) y_{n}=0 \\
& P(E) y_{n}=f_{n}
\end{aligned}
$$

Under the forward difference operator $\Delta$

$$
P(\Delta) y_{n}=0
$$

$$
P(\Delta) y_{n}=f_{n}
$$

Under the forward difference operator $\nabla$

$$
P(\nabla) y_{n}=0
$$

$$
P(\nabla) y_{n}=f_{n}
$$

where as $P$ is a polynomial.

For solving difference equations and finding general solution, we use the following theorems see [1], [2], [4], [5].

Theorem 1. (superposition principle) Suppose that $y_{n 1}, y_{n 2},, y_{n m}$ are the (fundamental) solutions of the homogeneous difference equation $P(\nabla) y_{n}=0$, then each arbitrary linear combination of them is a solution for it too.

Theorem 2. Suppose that the complex-valued function " $y_{n}=y_{n 1}+i y_{n 2}$ " is a solution of the homogeneous equation $P(\nabla) y_{n}=0$, then each of functions " $y_{n 1}, y_{n 2}$ " also are solutions for it.

Theorem 3. Let $y_{h}$ be a solution for the homogeneous $P(\nabla) y_{n}=0$ and $y_{p}$ be a solution for the nonhomogeneous $P(\nabla) y_{n}=f_{n}$, then " $y_{c}=y_{h}+y_{p}$ " is a solution for " $P(\nabla) y_{n}=f n "$ too. 


\section{Solution of the difference equations}

2.1. In this section, we discuss the difference equations with constant coefficients. Let " $y_{n}=r^{n}$ " be a solution for equations (1), (3) and (5), then $r^{n}$ must be satisfy in each of them, therefore we have, respectively

$$
\begin{gathered}
P(r)=0 \\
P(r-1)=0 \\
P\left(1-\frac{1}{r}\right)=0
\end{gathered}
$$

Where (7) is the corresponding characteristic equation to equation (1), and (8) is the corresponding characteristic equation to (3), and finally (9) is the corresponding characteristic equation to (5).

Remark 1. All roots of the characteristic equations may be distinct real values, either some of them are equal or some of them are conjugate complex number.

(i) If $r_{1}, r_{2}, r_{k}$ are distinct real roots to the characteristic equations, then $r_{1}^{n}, r_{2}^{n},, r_{k}^{n}$ will be solutions of homogeneous equations, these functions are linearly independent [3].

These functions are said fundamental solutions of homogeneous equation.

(ii) If $r_{1}=r_{2}=\ldots=r_{m}=r$, are the iterated root of the characteristic equation, then the corresponding solutions of homogeneous equation are: $" r^{n}, n r^{n}, n_{2} r^{n}, n_{m-1} r^{n} "$ that are linearly independent (see [3] ).

(iii) If $r_{12}=\alpha i \beta$, are two conjugate complex roots, in this case the corresponding solution of homogeneous equation are, " $y_{n 1}=\left(\alpha^{2}+\beta^{2}\right)^{\frac{n}{2}} \cos n \varphi, y_{n 2}=$ $\left(\alpha^{2}+\beta^{2}\right)^{\frac{n}{2}} \sin n \varphi$, where $\varphi=\tan ^{-1} \frac{\beta}{\alpha} "$ (see [3] ).

Example 1. Find the fundamental solutions of the following homogeneous difference equation

$$
\left(21 \nabla^{2}-10 \nabla+1\right) y_{n}=0 .
$$

Solution. We have "21(1- $\left.\frac{1}{r}\right)^{2}-10\left(1-\frac{1}{r}\right)+1=0 "$ which yields,

$$
" r_{1}=\frac{7}{6} \quad, \quad r_{2}=\frac{3}{2} \quad " \text { and } " \quad y_{n 1}=\left(\frac{7}{6}\right)^{n}, \quad y_{n 2}=\left(\frac{3}{2}\right)^{n} " .
$$


Example 2. Solve the following difference equation and find the fundamental solutions

$$
\left(\nabla^{3}-11 \nabla-150\right) y_{n}=0 .
$$

Solution. Roots of the corresponding characteristic equation are " $r_{1}=$ $\frac{-1}{5} \cdot r_{2,3}=\frac{1}{8}(1 \pm i)$ " in the result, then the fundamental solutions will be written as follows

$$
y_{n 1}=\left(\frac{-1}{5}\right)^{n} \quad, \quad y_{n 2}=2^{\frac{-5}{2} n} \cos \frac{n \pi}{4} \quad, \quad y_{n 3}=2^{\frac{-5}{2} n} \sin \frac{n \pi}{4}
$$

Lemma1. Prove the accuracy of the following equalities .

$$
\begin{aligned}
& \nabla \sum_{j=1}^{n} f_{j}=f_{n} . \\
& \frac{1}{\nabla} f_{n}=\sum_{j=1}^{n} f_{j} .
\end{aligned}
$$

Proof. Proof is easy, consider

$$
\nabla \sum_{j=1}^{n} f_{j}=\sum_{j=1}^{n} f_{j}-\sum_{j=1}^{n-1} f_{j}=f_{n} .
$$

Identity (11) is the inversion of (10).

Remark 2. Both of the above identities are used for finding the particular solution of the nonhomogeneous difference equations with constant coefficients, therefore we can solve each of the following equations

$$
\nabla y_{n}=f_{n} \quad, \quad \nabla^{m} y_{n}=f_{n} .
$$

Example 3. Find the particular solution of the following difference equation

$$
\nabla y_{n}=n^{3}-n^{2}+2 n+2 .
$$


Solution. We can write

$y_{p}=\frac{1}{\nabla}\left(n^{3}-n^{2}+2 n+2\right)=\sum_{j=1}^{n}\left(j^{3}-j^{2}+2 j+2\right)=\frac{1}{12} n\left(3 n^{3}+2 n^{2}+3 n+28\right)$.

Example 4. Find the particular solution of the following difference equation

$$
\nabla^{3} y_{n}=120 n+60
$$

Solution. By division operation we can write

$$
\begin{gathered}
y_{p}=\frac{1}{\nabla^{3}}(120 n+60)=\frac{1}{\nabla^{2}}\left(\frac{1}{\nabla}(120 n+60)\right) \\
=\frac{1}{\nabla^{2}}\left(\sum_{j=1}^{n}(120 j+60)\right)=\frac{1}{\nabla}\left(\frac{1}{\nabla}\left(60\left(n^{2}+2 n\right)\right)\right) \\
=\frac{1}{\nabla}\left(\sum_{j=1}^{n} 60\left(j^{2}+2 j\right)=\frac{1}{\nabla}\left(10\left(2 n^{3}+9 n^{2}+7 n\right)\right)\right. \\
=10 \sum_{j=1}^{n}\left(2 j^{3}+9 j^{2}+7 j\right)=5 n(n+1)(n+3)(n+4) .
\end{gathered}
$$

Example 5. Find the particular solution of the following difference equation

$$
\nabla \Delta y_{n}=\cos \frac{n \pi}{3}
$$

Solution. Since $\Delta \equiv E \nabla$ we can write

$$
\begin{gathered}
y_{p}=\frac{1}{E \nabla^{2}}\left(\cos \frac{n \pi}{3}\right)=\frac{1}{\nabla^{2}} \cos \frac{(n-1) \pi}{3} \\
=\frac{1}{\nabla} \sum_{j=0}^{n-1} \cos \frac{(j-1) \pi}{3}=\sum_{j=0}^{n-1}\left(\frac{-1}{2}+\sin \frac{(2 j-1) \pi}{6}\right)=1-\frac{1}{2} n-\cos \frac{n \pi}{3} .
\end{gathered}
$$

\section{Main Results}

\subsection{Backward $r$-difference operators and solution of nonhomoge- neous difference equations}


Definition. We define the backward $r$-difference operator $\Delta_{r}$ as follows

$$
\nabla_{r} y_{n}=y_{n}-r y_{n-1}=\left(1-r E^{-1}\right) y_{n}
$$

where $y_{n}$ is the approximation of function $y(x)$ at point $x_{n} \in\left[x_{0}, x_{m}\right]$, then two operators " $\nabla_{r}$ " and " $1-r E^{-1}$ " are equivalent.

Corollary 1. $\nabla_{r}$ is linear operator and $\nabla_{1}=\nabla=1-E^{-1}$.

Example 6. $\quad \nabla_{r}\left(r^{n} y_{n}\right)=r^{n} y_{n}-r r^{n-1} y_{n-1}=r^{n}\left(y_{n}-y_{n-1}\right)=r^{n} \nabla y_{n}$ $\nabla_{4}\left(4^{n} \cos \frac{n \pi}{2}\right)=4^{n} \nabla \cos \frac{n \pi}{2}=4^{n}\left(\cos \frac{n \pi}{2}-\cos \frac{n \pi}{2}\right)$.

Example 7.

$\nabla_{5}\left(5^{n}\left(n^{2}-6 n+10\right)\right)=5^{n}\left(n^{2}-6 n+10\right)-55^{n-1}\left((n-1)^{2}-6(n-1)+10\right)=5^{n}(2 n-1)$.

\section{Four principal operations in vector space of the operator $r$ :}

we define

$$
\begin{aligned}
& \nabla_{r}+\nabla_{s} \equiv 2 \nabla_{\frac{r+s}{2}} . \\
& \nabla_{r}-\nabla_{s} \equiv(s-r) E^{-1} \equiv \nabla_{r-s}-1 . \\
& \nabla_{r} \nabla_{s} \equiv 1-(s+r) E^{-1}+r s E^{-2} \\
& \frac{\nabla_{r}}{\nabla_{s}} \equiv \nabla_{r} \frac{1}{\nabla_{s}} \equiv \nabla_{s} \frac{1}{\nabla_{r}}
\end{aligned}
$$

Particular type 1. Suppose that $s=r$, then

$$
\nabla_{r} \nabla_{r} \equiv \nabla_{r}^{2}, \quad \cdots \quad, \quad \nabla_{r} \nabla_{r}^{m} \equiv \nabla_{m+1}
$$

In the sequel, we define order and inversion for the backward $r$-difference operator.

$$
\begin{gathered}
\nabla_{r}^{-1} \equiv \frac{1}{\nabla_{r}} \quad \text { s.t } \quad \frac{1}{\nabla_{r}} f_{n}=g_{n} \Leftrightarrow \nabla_{r} g_{n}=f_{n} \\
\nabla_{r}^{2} \equiv \nabla_{r}\left(\nabla_{r}\right), \ldots, \nabla_{r}^{m+1} \equiv\left(\nabla_{r}^{m}\right)
\end{gathered}
$$

Remark 3. Addition operation and multiplication operation have two properties: commutative and associative, namely

$$
\left(\nabla_{r}+\nabla_{s}\right)+\nabla_{t} \equiv \nabla_{r}+\left(\nabla_{s}+\nabla_{t}\right) \equiv 3 \nabla_{\frac{r+s+t}{3}}, \quad \nabla_{r}\left(\nabla_{s} \nabla_{t}\right) \equiv\left(\nabla_{r} \nabla_{s}\right) \nabla_{t}
$$


Theorem 4. The backward $r$-difference is linear operator, in addition to every order of it and every polynomial of $\nabla_{r}$ and inversion $\nabla_{r}^{-1}$ are linear too. Proof. It is easy and left to the readers.

Lemma2. Prove that

$$
\begin{aligned}
& \nabla_{r}\left(\sum_{j=1}^{n} r^{n-j} y_{j}=y_{n} .\right. \\
& \frac{1}{\nabla_{r}} y_{n}=\left(\sum_{j=1}^{n} r^{n-j} y_{j} .\right.
\end{aligned}
$$

Proof. It is easy. Two above formulas are used in finding solution of NDE.

Remark 4. Under the backward $r$-difference operator $\nabla_{r}$, the nonhomogeneous difference equation is written in the following form

$$
\left(\prod_{j=1}^{m} \nabla_{r_{j}}\right) y_{n}=f_{n}
$$

Whereas " $r_{j}, j=1,2, \ldots, m$ " can be real distinct, iterated or complex number.

Some useful results $(a \neq r)$

$$
\begin{aligned}
& \nabla_{r}\left(a^{n}\right)=a^{n-1}(a-r) \Rightarrow \frac{1}{\nabla_{r}} a^{n}=\frac{a^{n+1}}{a-r} \\
& \nabla_{r}^{k}\left(a^{n}\right)=a^{n-k}(a-r)^{n-k} \Rightarrow \frac{1}{\nabla_{r}^{k}} a^{n}=\frac{a^{n+k}}{(a-r)^{k}} \\
& \nabla_{r}\left(n a^{n}\right)=n a^{n}-r(n-1) a^{n-1}=(n a-r(n-1)) a^{n-1} \\
& \Rightarrow \frac{1}{\nabla_{r}}\left(n a^{n}\right)=\frac{n(a-r)-r}{(a-r)^{2}} a^{n+1} . \\
& P\left(\nabla_{r}\right) a^{n}=P\left(1-\frac{r}{a}\right) a^{n} \Rightarrow \frac{1}{P\left(\nabla_{r}\right)} a^{n}=\frac{a^{n}}{P\left(1-\frac{r}{a}\right)}
\end{aligned}
$$

Example 8. $\frac{1}{\nabla_{2}}\left(n^{2} 5^{n}\right)=\left(\frac{9 n^{2}-12 n+14}{27}\right) 5^{n+1}$.

Lemma 3. Let $m$ be integer and $a \neq r$ then

$$
\begin{aligned}
& \nabla_{r}^{m}\left(a^{n} y_{n}\right)=a^{n} \nabla_{\frac{r}{a}}^{m} y_{n} . \\
& \frac{1}{\nabla_{r}^{m}}\left(a^{n} y_{n}\right)=a^{n} \frac{1}{\nabla_{\frac{r}{a}}^{m}} y_{n} .
\end{aligned}
$$


Proof. Equality (15) is proved by the mathematical induction, (16) is the inversion of (14).

Particular type 2. Let $a=r$, then last equalities will be as follows, respectively:

$$
\begin{aligned}
& \nabla_{r}^{m}\left(r^{n} y_{n}\right)=a^{n} \nabla^{m} y_{n} . \\
& \frac{1}{\nabla_{r}^{m}}\left(r^{n} y_{n}\right)=r^{n} \nabla^{m} y_{n} .
\end{aligned}
$$

Example 9. Evaluate $\frac{1}{\nabla_{2}^{3}}\left(2^{n} n\right)$.

Solution. $\quad \frac{1}{\nabla_{2}^{3}}\left(2^{n} n\right)=2^{n} \frac{1}{\nabla^{3}}(n)=2^{n} \frac{1}{\nabla^{2}} \sum_{j=1}^{n} j=2^{n-1} \frac{1}{\nabla}\left(\sum_{j=1}^{n}\left(j^{2}+1\right)\right)$.

$$
\frac{2^{n-1}}{3} \sum_{j=1}^{n}\left(j^{3}+3 j^{2}+2 j\right)=\frac{2^{n-3}}{3} n(n+1)(n+2)(n+3) \text {. }
$$

Theorem 5 (shift exponential). Let $P$ be a polynomial, then

$$
\begin{aligned}
& P\left(\nabla_{r}\right)\left(a^{n} y_{n}\right)=a^{n} P\left(\nabla_{\frac{r}{a}}\right) y_{n} . \\
& \frac{1}{P\left(\nabla_{r}\right)}\left(a^{n} y_{n}\right)=a^{n} \frac{1}{P\left(\nabla_{\frac{r}{a}}\right)} y_{n} .
\end{aligned}
$$

Proof. It is easy and possible by Lemma2 .

Particular type 3. Suppose that $a=r$, then the former equalities may be written in the following forms

$$
\begin{aligned}
& P\left(\nabla_{r}\right)\left(r^{n} y_{n}\right)=r^{n} P(\nabla) y_{n} . \\
& \frac{1}{P\left(\nabla_{r}\right)}\left(r^{n} y_{n}\right)=r^{n} \frac{1}{P(\nabla)} y_{n} .
\end{aligned}
$$


Example 10. Find the particular solution of the following NDE

$$
\left(E^{4}-10 E^{3}+35 E^{2}-50 E+24\right) y_{n}=2^{n}(8 n+12) .
$$

Solution. This equation may be written as follows :

$$
\begin{gathered}
(E-1)(E-2)(E-3)(E-4) y_{n}=E^{4} \nabla \nabla_{2} \nabla_{3} \nabla_{4} y_{n} \\
=\frac{1}{4} E^{4}\left(1+\nabla_{2}\right) \nabla_{2}\left(-1+3 \nabla_{2}\right)\left(-1+2 \nabla_{2}\right) y_{n}=(8 n+12) 2^{n} .
\end{gathered}
$$

Now after dividing two sides of this equality by the coefficient of $y_{n}$, using the formula (22), we have

$$
\begin{aligned}
y_{p} & =\frac{1}{\left(1+\nabla_{2}\right) \nabla_{2}\left(-1+3 \nabla_{2}\right)\left(-1+\nabla_{2}\right)}(2 n-5) 2^{n-2} \\
& =2^{n-2} \frac{1}{(1+\nabla) \nabla(-1+3 \nabla)(-1+\nabla)}(2 n-5)=2^{n}(n+4) .
\end{aligned}
$$

\subsection{Solution of NDE with constant coefficients}

We know that every nonhomogeneous difference equation with order $\mathrm{m}$ can be written in the form (14).

Therefore each of the following forms may be written in the form in (14).

$$
P(E) y_{n}=f_{n} \quad, \quad P(\Delta) y_{n}=f_{n} \quad, \quad P(\nabla) y_{n}=f_{n} .
$$

(14) is written as follows.

$$
y_{p}=\frac{1}{\prod_{j=1}^{m} \nabla_{r_{j}}} f_{n}=\frac{1}{\nabla_{r_{m}}}\left(\frac{1}{\nabla_{r_{m-1}}}\left(\ldots \frac{1}{\nabla_{r_{1}}} f_{n} \ldots\right)\right) .
$$

Example 11. Find the particular solution of $\nabla^{2} \nabla_{2} \nabla_{3} y_{n}=n 2^{n}$.

Solution. We have

$$
\begin{gathered}
y_{p}=\frac{1}{\nabla^{2} \nabla_{2} \nabla_{3}}\left(n 2^{n}\right)=\frac{1}{\nabla_{2}}\left(\frac{1}{\nabla_{3}}\left(\frac{1}{\nabla^{2}}\left(n 2^{n}\right)\right)\right)=\frac{1}{\nabla_{2}}\left(\frac{1}{\nabla_{3}}\left(\frac{1}{\nabla}(2 n-2) 2^{n}\right)\right. \\
=\frac{1}{\nabla_{2}}\left(\frac{1}{\nabla_{3}}(4 n-8) 2^{n}\right)=\frac{1}{\nabla_{2}}\left((-2 n+1) 2^{n}\right)=-n^{2} 2^{n} .
\end{gathered}
$$


Remark 5. In using identity (23), we may use iterative divisions, in addition to, we can use the decomposition fraction, consider

$$
\begin{gathered}
\frac{1}{\nabla_{r_{1}} \nabla_{r_{2}}} \equiv \frac{1}{r_{1}-r_{2}}\left(\frac{r_{1}}{\nabla_{r_{1}}}-\frac{r_{2}}{\nabla_{r_{2}}}\right) \\
\frac{1}{\nabla_{r_{1}} \nabla_{r_{2}} \nabla_{r_{3}}} \equiv \frac{r_{1}^{2}}{\left(r_{1}-r^{2}\right)\left(r_{1}-r_{3}\right)} \frac{1}{\nabla_{r_{1}}}+ \\
\frac{r_{2}^{2}}{\left(r_{2}-r^{1}\right)\left(r_{2}-r_{3}\right)} \frac{1}{\nabla_{r_{2}}}+\frac{r_{3}^{2}}{\left(r_{3}-r^{1}\right)\left(r_{3}-r_{2}\right)} \frac{1}{\nabla_{r_{3}}} .
\end{gathered}
$$

In general $\frac{1}{\prod_{j=1}^{m} \nabla r_{j}} \equiv \sum_{j=1}^{m} \frac{A_{j}}{\nabla r_{j}}$ where,

$$
\sum_{j=1}^{m} A_{j}=1, A_{j}=\frac{r_{j}^{m-1}}{\prod_{j \neq i=1}^{m}}\left(r_{j}-r_{i}\right)
$$

Example 12. Find the particular solution of the following NDE

$$
\nabla_{2} \nabla_{4} y_{n}=n 2^{n}
$$

Solution.

$$
\begin{aligned}
y_{p} & =\frac{1}{\nabla_{2} \nabla_{4}}\left(n 2^{n}\right)=\frac{1}{\nabla_{4}}\left(n 2^{n+1}\right)-\frac{1}{\nabla_{2}}\left(n 2^{n}\right) \\
& =-(n+2) 2^{n+1}-2^{n-1}\left(n^{2}+n\right)=-2^{n-1}\left(n^{2}+5 n+8\right) .
\end{aligned}
$$

Example 13. Find the particular solution of the following NDE

$$
\left(36-36 E^{-1}+11 E^{-2}-E^{-3}\right) y_{n}=6^{n-3}(6 n 2-48 n+80) .
$$

Solution. Write $\left(36 E^{3}-36 E^{2}+11 E-1\right) y_{n}=6^{n}\left(6 n^{2}-12 n-10\right)$.

$$
\begin{gathered}
y_{p}=\frac{1}{(2 E-1)(3 E-1)(6 E-1)}\left(6^{n}\left(6 n^{2}-12 n-10\right)\right) \\
=\frac{1}{36 \Delta_{\frac{1}{2}} \Delta_{\frac{1}{3}} \Delta_{\frac{1}{6}}}\left(6^{n}\left(6 n^{2}-12 n-10\right)\right) 6^{n+1} \frac{1}{\Delta_{\Delta_{2} \Delta_{3}}}\left(6 n^{2}-12 n-10\right) \\
=6^{n+1}\left(\frac{\frac{1}{2}}{\Delta}-\frac{1}{\Delta_{2}}+\frac{\frac{1}{2}}{\Delta_{3}}\right)\left(6 n^{2}-12 n-10\right)=6^{n+1} n^{3} .
\end{gathered}
$$


Remark 6. If $r_{1}=r_{2}=\ldots=r_{m}=r$, then we can use only iterative divisions.

Remark 7. Let $P\left(\Delta_{r}\right) y_{n}=a^{n} f_{n}$, then by change of variable " $y_{n}=Y_{n} a^{n}$ " and using the shift exponential formula, we obtain the following NDE.

$$
P\left(a \nabla_{\frac{r}{a}}\right) Y_{n}=f_{n}
$$

Remark 8. Let $P\left(\nabla_{r}\right) y_{n}=a^{n}(a \neq 1)$,

(i) If $P\left(1-\frac{r}{a}\right) \neq 0$, then

$$
y_{p}=\frac{1}{P\left(1-\frac{r}{a}\right)} a^{n}
$$

(ii) If $P\left(1-\frac{r}{a}\right)=P^{\prime}\left(1-\frac{r}{a}\right)=\ldots=P^{(k-1)}\left(1-\frac{r}{a}\right)=0, P^{(k)}\left(1-\frac{r}{a}\right) \neq 0$, then

$$
y_{p}=\frac{1}{r^{k} P^{(k)}\left(1-\frac{r}{a}\right)}\left(n^{k} a^{n+k}\right) .
$$

\section{Concluding.}

The backward r-difference operator method is a new method in finding solution of the nonhomogeneous difference equations with constant coefficients and we can use it in finding solution of the following equation

$$
P(\nabla) y_{n}=f_{n}
$$

where

$$
P(\nabla) \equiv \sum_{j=0}^{m} a_{j} \nabla_{j}
$$

$a_{j}$ 's are constants .

\section{$5 \quad$ References}

[1] Hildel brand, F.B., "Introduction to Numerical Analysis", Mc. GrawHill, New.York, 1956.

[2] Isaacson, E. and H.B., "Analysis of Numerical Methods", John Wiley and Sons,..New York, 1966. 
[3] Lambert, J.D. "Computation methods in ordinary Differential Equations" John .Wiley and Sons, New York, 1973.

[4] Phillips, G.M., Taylor P.J. "Theory and Applications of Numberical Analysis", Fifth. Edition, Academic press, 1980.

[5] Ralston, A. "A first course in Numerical Analysis "Mc. Graw-Hill, New York.

Received: November 8, 2006 